\title{
EFICIENCIA DE LA PROTECCIÓN CONTRA INCENDIOS FORESTALES EN MONTE ALEGRE, BRASIL Y PINAR DEL RÍO, CUBA
}

\author{
Marcos Pedro Ramos Rodríguez ${ }^{1}$, Ronaldo Viana Soares ${ }^{2}$, Antonio Carlos Batista ${ }^{2}$, \\ Alexandre França Tetto ${ }^{2}$, Jesús María Cabrera Reina ${ }^{3}$, Luis Wilfredo Martínez Becerra ${ }^{4}$ \\ ${ }^{1}$ Ing. Forestal, Dr.C., Dpto. Forestal, UPR, Pinar Del Rio, Cuba - cmramos@af.upr.edu.cu \\ ${ }^{2}$ Ing. Forestal, Dr., Dpto. de Ciencias Forestales, UFPR, Curitiba, Brasil - rvsoares@ufpr.br; batistaufpr@ufpr.br; tetto@ufpr.br \\ ${ }^{3}$ Ing. Forestal, M.Sc., Dpto. Manejo del Fuego, CGB, Pinar Del Rio, Cuba - pinar@cgb.rem.cu \\ ${ }^{4}$ Ing. Forestal, Dr.C., Dpto. Forestal, UPR, Pinar Del Rio, Cuba - wmartinez@af.upr.edu.cu \\ Recebido para publicação: 20/08/2012 - Aceito para publicação: 05/04/2014
}

\begin{abstract}
Resumen
Para perfeccionar el sistema de protección contra incendios forestales es importante tener informaciones sobre su eficiencia. Este trabajo fue desarrollado con el objetivo de comparar la eficiencia del sistema de protección contra incendios forestales en la Fazenda Monte Alegre, estado del Paraná, sur de Brasil y en las Empresas Forestales de la Provincia Pinar del Río, Cuba. Los datos utilizados se refieren al período 01/01/2001 - 31/12/2010. Para evaluar la eficiencia fueron utilizados indicadores tales como: densidad de incendios, densidad de área quemada, áreas quemadas medias por incendios, porcentaje del área forestal quemada y tamaño de los incendios. Los indicadores fueron estimados considerando la distribución temporal y espacial de los incendios. De forma general los valores medios obtenidos para los indicadores muestran mejor eficiencia en Monte Alegre. En ambas regiones la eficiencia fue menor durante los meses correspondientes a la época de incendios. En Monte Alegre la eficiencia fue similar en bosques naturales y en plantaciones, mientras que en Pinar del Río es menor en los bosques naturales. La eficiencia obtenida para el sistema de protección contra incendios tanto en Monte Alegre como en Pinar del Río, es menor en el grupo de especies de los Pinus spp.

Palabras-clave: Prevención; combate; densidad de incendios; densidad de área quemada; clases de tamaño.
\end{abstract}

\begin{abstract}
Forest fire protection efficiency in Monte Alegre, Brazil and Pinar del Río, Cuba. It's important to have information about the efficiency of the forest fire protection system to take decisions that will improve the protection against wildfires. The objective of this work was to compare the efficiency of the forest fire protection system in the Monte Alegre Forest District, State of Parana, south of Brazil and the Forest Companies of Pinar del Río Province, Cuba. A ten year period (01/01/2001 to 31/12/2010) was considered. For the efficiency evaluation, we used the following indicators: forest fires density, burned area density, burned area average by fire, burned surface percentage, and fire size class. These indicators have been considered observing temporary and spatial distribution of forest fire. After analyzing all the indicators, results revealed a higher efficiency regarding fire control in the Monte Alegre fire protection system. In both regions, the efficiency was lower in the fire season. In Monte Alegre, the efficiency was similar in natural forests and plantations. However, in Pinar del Río, the efficiency was lower in natural forests. The efficiency of forest fire protection system in Monte Alegre and Pinar del Río was lower in the Pinus spp plantations. Keywords: Forest fires; prevention; suppression; fire size; forest protection.
\end{abstract}

\section{INTRODUCCIÓN}

Las dos actividades clásicas de un sistema de protección contra incendios forestales son la prevención y la extinción. A través de la prevención se reducen o eliminan tanto las fuentes de fuego de naturaleza humana, como las fuentes de propagación del fuego. Se consigue de esta forma reducir las ocurrencias y dificultar la propagación del fuego. A través de la extinción se evita que los incendios 
iniciados, quemen grandes áreas. Estas actividades se desarrollan muy interrelacionadas, por esto es más común evaluar la eficiencia del sistema de protección en su conjunto.

Los incendios forestales se caracterizan por altos niveles de destrucción del capital natural y humano, imponiendo una gran capacidad financiera, económica, carga humana y del medio ambiente en las sociedades mediterráneas. La incidencia y el alcance de los incendios forestales son inciertos, dependiendo fundamentalmente de dos factores: la probabilidad de del inicio de los incendios y la existencia de condiciones que favorecen su propagación prevaleciente. La única manera de reducir el número y intensidad de los incendios forestales y reducir el daño económico es implementar programas de prevención y combate (MENDES, 2010).

Los incendios forestales son muy caros para combatir y pueden quemar miles de hectáreas de bosques si no se puede controlar, siendo que algunas veces resultan en pérdidas de vidas humanas. Cuando un ataque inicial fuerte y rápido es realizado, la mayoría de los incendios son impedidos de crecer de manera descontrolada, causando daños ambientales. Mientras tanto, se un ataque inicial fallar, entonces el fuego sale del control y puede causar daños independiente de su tamaño. Los gestores y planificadores de los incendios, muchas veces disponen de pocos recursos, limitaciones presupuestarias y la incertidumbre acerca de la ocurrencia y el comportamiento del fuego. Por lo tanto, es necesario determinar si un incendio en particular puede o no ser contenido (NTAIMO et al., 2012).

Un sistema de monitoreo, predicción y contención exitosa de incendios forestales debe basarse en la existencia y el funcionamiento eficaz de un sistema de seguimiento bien organizado y equipado y mecanismos de supresión. El daño excesivo causado por los incendios forestales a nivel mundial es una prueba contundente de la necesidad de tal mecanismo. Las características esenciales de cualquier sistema que tiene como objetivo hacer frente con eficacia al problema de la extinción de incendios forestales son los modelos de predicción de incendios, junto con las observaciones de campo y modelos para el despliegue óptimo de los recursos disponibles (RACHANIOTIS; PAPPIS, 2006).

Una parte crítica de la utilización de un enfoque de respuesta estándar para planificación de incendios forestales de ataque inicial es determinar lo que constituye una "respuesta estándar". Esto se hace generalmente sobre la base del tamaño esperado del fuego y el número de recursos de extinción dentro de un plazo máximo recorrido (distancia) que contendría el fuego (HAIGHT; FRIED, 2007).

Bajo el punto de vista de la teoría microeconómica, la lucha contra los incendios forestales puede ser vista como una actividad productiva, cuya salida, medida en alguna unidad física por unidad de tiempo (por ejemplo, $x$ número de hectáreas potencialmente salvadas durante el período de tiempo $t$ quemado; o el diseño de una línea controlada de fuego de $x$ metros durante el período de tiempo $y$, o el número $x$ de la quema de hectáreas a extinguirse por período de tiempo, etc.) depende: i) del tipo y cantidad de insumos materiales y humanos; y ii) de la forma en que se combinan estos insumos, es decir, las actividades de lucha contra los incendios dependen de la respectiva tecnología desplegada (MENDES, 2010).

Para esto pueden utilizarse varios indicadores. Algunos de ellos han sido utilizados como parte de las evaluaciones del comportamiento histórico de los incendios forestales en Brasil (SOARES, 1988, 2009; TETTO et al., 2012a), en España (VÉLEZ, 1990; DIRECCIÓN GENERAL DE CONSERVACIÓN DE LA NATURALEZA (DGCN), 1996; COMITÉ DE LUCHA CONTRA INCENDIOS FORESTALES (CLIF), 1997), en Turquía (CANAKÇIOGLU, 1990), en Cuba (RODRÍGUEZ, 1999), en Australia (McCARTHY et al., 2012), en Brasil y Cuba (RODRÍGUEZ; SOARES, 2004; RODRÍGUEZ et al., 2008 e 2013). El indicador más común, y generalmente el único que se usa, es la media del área quemada por incendio anualmente. Esto puede enmascarar determinados resultados y dificultar el análisis. El segundo lugar en cuanto a su utilización lo ocupa el indicador tamaño de los incendios. La utilización de indicadores tales como: densidad de incendios, densidad de área quemada, media del área quemada por incendio, y porcentaje de la superficie boscosa que se quema, han sido encontrados en Rodríguez (1999), Rodríguez y Soares (2004), y Rodríguez et al. (2008). Lima y Soares (1992) evaluaron la eficiencia del combate a través de los indicadores: tiempo de movilización, tiempo de combate, número de combatientes, y número de horas-hombre por hectárea quemada. Tetto et al. (2012b) evaluaron la eficiencia por medio de los indicadores: clases de tamaño dos incendios, área quemada y tiempo gasto entre la detección y la extinción del fuego.

La clasificación de los incendios por clases de tamaño es un dato importante para evaluar la efectividad del servicio de combate de incendios, pues mientras mayor sea esta efectividad, mayor será la concentración de los incendios en las clases de menor área (SOARES, 1985; TETTO et al., 2012b). También en el resto de los indicadores los valores pequeños indican mayor eficiencia.

Normalmente los indicadores anteriores se analizan únicamente para un determinado periodo de 
tiempo. No obstante, como los incendios forestales son un fenómeno espacio-temporal, es importante hacer cada análisis precisamente en estos dos contextos, lo cual permitirá definir con mejor precisión cuándo y dónde el sistema de protección es más o menos eficiente. Debido a esto Rodríguez (1999) plantea que los análisis deben observar la distribución temporal (periodo de años seleccionado y meses del año) y espacial (tipo de cobertura forestal y grupos de especies) de los incendios.

Considerando que las informaciones sobre la eficiencia del sistema de protección contra incendios contribuyen al perfeccionamiento de las actividades de manejo del fuego, se probó la hipótesis de que la eficiencia del sistema de protección contra incendios es diferente en las dos regiones estudiadas. La comparación de dos regiones ubicadas en distintos hemisferios, con climas y sistemas socioeconómicos diferentes trata de determinar la existencia de posibles similitudes y diferencias en cuanto a la manifestación de la eficiencia de la protección contra incendios forestales. Los resultados deben permitir reflexionar en dos sentidos, es decir, los que son similares y permiten observar que dos regiones manifiestan resultados similares demostrando que existen regularidades asociadas al fenómeno de los incendios forestales que en definitiva es un problema global. Si los resultados son diferentes pues entonces la idea es hacer los análisis necesarios con el fin de que en el lugar donde sean malos o regulares, lleguen a ser buenos o excelentes. Si los resultados son buenos en ambos lugares, entonces puede buscarse el porqué. En correspondencia con esto el objetivo de este trabajo fue comparar la eficiencia del sistema de protección contra incendios forestales en la Fazenda de Monte Alegre, estado del Paraná, sur de Brasil y en las Empresas Forestales de la Provincia Pinar del Río, Cuba, durante un periodo de 10 años (2001-2010).

\section{MATERIAL Y MÉTODOS}

\section{Caracterización del área de estudio}

El trabajo se desarrolló en dos regiones:

- La Fazenda Monte Alegre, estado del Paraná, sur de Brasil, que tiene un área total de $1.312 \mathrm{~km}^{2}$ y está situada entre $\operatorname{los} 24^{\circ} 03^{\prime}$ y $24^{\circ} 31^{\prime}$ de latitud sur y entre $\operatorname{los} 50^{\circ} 10^{\prime}$ y $50^{\circ} 50^{\prime}$ de longitud oeste. El relieve de la región es suavemente ondulado y el más importante accidente geográfico es el río Tibagi, principal afluente del río Paranapanema, que por su vez es uno de los principales formadores del río Paraná. La altitud media del área de estudio es de $850 \mathrm{~m}$ s.n.m., con amplitud entre 750 y 868 m (SOARES, 1972; NUNES et al., 2009 e 2010). La precipitación anual en la región es de 1399,5 $\mathrm{mm}$, la temperatura media anual de $19^{\circ} \mathrm{C}$ y la humedad relativa media anual de $76,5 \%$. Según Maack (1968), de acuerdo con la clasificación de Köppen, la región pertenece al tipo Cfb (clima templado con precipitaciones uniformes a lo largo del año y verano caliente, con 4 o más meses con temperatura media mensual del mes más caliente por encima de $10{ }^{\circ} \mathrm{C}$ ).

- La provincia de Pinar del Río, se encuentra situada en el extremo oeste de la isla de Cuba, con una superficie de $10.901 \mathrm{~km}^{2}$, está situada entre los $21^{\circ} 45^{\prime}$ y $23^{\circ} 01^{\prime}$ de latitud norte y entre los $82^{\circ} 51^{\prime}$ y $84^{\circ} 57^{\prime}$ de longitud oeste. La precipitación media anual de la región es de $1.484,53 \mathrm{~mm}$, la temperatura media anual de $24,5{ }^{\circ} \mathrm{C}$ y la humedad relativa media anual de $81 \%$. Según Wadsworth (2000) de acuerdo con la clasificación de Köppen el clima es Aw (clima tropical con mes más frío con temperatura mayor de $18{ }^{\circ} \mathrm{C}$ y una estación seca de al menos un mes con menos de $600 \mathrm{~mm}$ ). La provincia de Pinar del Río es atravesada por la Cordillera de Guaniguanico la cual según el Instituto de Planificación Física (IPF) (1993) constituye uno de los tres macizos montañosos más importantes de Cuba por su extensión, altura y configuración, extendiéndose con orientación ENE a WSW a lo largo de casi todo el territorio de la provincia de Pinar del Río y abarca un área de $3625,4 \mathrm{~km}^{2}$, el 33,46\% de la superficie de la misma. La integran la Sierra del Rosario en la porción centro-este con un área de $923,4 \mathrm{~km}^{2}$ (el 25\%) y la Sierra de los Órganos en la parte centro-oeste con dos sistemas fisiográficos fundamentales: las Alturas de Pizarras del Norte y del Sur, con superficie de $2.702,0 \mathrm{~km}^{2}$ (el $75 \%$ del área de la cordillera). En esta cordillera se manifiestan interesantes combinaciones de premontañas, montañas, mesetas, mogotes y valles que la tipifican, destacándose como sus unidades fisiográficas más importantes sus dos mayores elevaciones: Pan de Guajaibón con 699 m s.n.m. y Cerro de Cabras con $484 \mathrm{~m}$ s.n.m. Puede decirse que el relieve de la zona de estudio favorece la velocidad de propagación del fuego y dificulta las acciones de combate, siendo la pendiente el factor de la topografía de mayor importancia desde el punto de vista de su acción sobre el comportamiento del fuego. Puede agregarse que un porcentaje alto de los caminos, por su mal estado, dificultan y en algunos casos no permiten, el acceso hasta los principales macizos boscosos ubicados en las zonas montañosas. 
En Pinar del Río para la vigilancia se cuenta con siete torres de observación, un avión AN-2 que se contrata para la época de incendios (febrero-mayo) y guardabosques de patrullaje que en horas de máxima ocurrencia de incendios se detienen en puntos elevados del terreno. La vigilancia desde las torres se realiza todo el año. La permanencia en las mismas depende del índice de peligro diario y las horas de mayores ocurrencias. Los guardabosques de patrullaje realizan su trabajo durante todo el año y utilizando caballos para el traslado. Los itinerarios a recorrer se establecen en dependencia del plan de vigilancia y protección. Además de lo anterior, durante la época de incendios se activan 28 puntos de observación atendidos por fuerzas del Cuerpo de Guardabosques (CGB). Durante la época de incendios también se activan 173 puntos que son atendidos por fuerzas eventuales, es decir, personal contratado durante la época de peligro, con los cuales pueden activarse también puntos de control en caminos que permiten el acceso al bosque. También se cuenta con información sobre focos de calor detectados por el satélite GOES.

El sistema de alerta en la provincia está constituido por la determinación diaria del índice de peligro Fórmula de Monte Alegre (FMA) en cada una de las Brigadas Profesionales de Prevención y Combate a los Incendios Forestales (BPPCIF). También en la Jefatura Provincial del CGB se cuenta con informaciones sobre la lluvia caída en las últimas 24 horas y su acumulado, además de los pronósticos sobre su posible comportamiento en los próximos siete días. También se analiza el pronóstico sobre velocidad y dirección de los vientos que emite el Instituto de Meteorología. Además, calculan el índice de peligro FMA para los próximos 7 días utilizando datos disponibles en la internet.

Para el combate a los incendios forestales en la provincia se cuenta con un total de nueve BPPCIF. Todas estas brigadas cuentan para el traslado de sus fuerzas con un vehículo ligero de doble tracción en el cual se trasladan también tanto las herramientas manuales de combate como bombas de espalda, antorchas de goteo y motosierras. La mayoría de estas brigadas cuenta con camiones de bomberos con capacidad aproximada de 1200 litros de agua. Adicionalmente dos brigadas cuentan con sistemas de bombeo de agua formado por mangueras y motobombas MAK-3 que permiten llevar el agua hasta una distancia aproximada de $1 \mathrm{~km}$. Además de las BPPCIF en la provincia se encuentran organizadas un total de 31 Brigadas Especializadas de Combate a los Incendios Forestales. Estas brigadas se organizan en las entidades propietarias de los bosques y cuentan con herramientas agrícolas y medios de transporte. Adicionalmente existen 11 Brigadas Voluntarias de Protección al Bosque y la Fauna. Todas las brigadas son entrenadas y capacitadas todos los años. Es importante destacar que las comunicaciones entre los distintos elementos que forman el sistema de protección están garantizadas y que los incendios han sido declarados desastres, motivo por el cual se encuentra organizada la cooperación entre las distintas entidades que forman el sistema de la Defensa Civil, la cual es activada en el momento en que se pronostica que el incendio va a alcanzar grandes proporciones.

El sistema de detección en la Fazenda Monte Alegre es compuesto de 7 torres de observación situadas em las siguientes regiones forestales: Cerradinho, Vila Preta, Agronomia, Anta Brava, Boa Esperança, Fazenda Velha e Pinhal Bonito, siendo que el recobrimiento eficaz de la región fue objeto de investigación de Venturi y Antunes (2007). De la misma manera que en Pinar del Río, la permanencia en las mismas depende del índice de peligro diario (calculado diariamente para a área) y las horas de mayores ocurrencias.

Son utilizados para el combate dos camiones bomberos ubicados en Lagoa (Boa Esperança) y en el municipio de Telêmaco Borba. Además, existe una camioneta para disminuir el tiempo del primero combate, con cuatro combatientes (siendo un el líder de campo) y equipada con dos bombas de espalda, dos bate-fuegos, dos azadas y un rozón. El sistema cuenta también con tres vigilantes motorizados y operadores de las torres también motorizados.

\section{Proceso metodológico}

Los datos relacionados con los incendios forestales fueron suministrados por el Área de Protección Patrimonial de la Klabin S/A, Monte Alegre, Brasil y por el Cuerpo de Guardabosques de la Provincia Pinar del Río, Cuba. Los datos relacionados con la dinámica forestal fueron suministrados por la Klabin S/A, Monte Alegre, Brasil y por el Servicio Estatal Forestal de la provincia Pinar del Río. Todos los datos se refieren al período 01/01/2001 - 31/12/2010, totalizando 10 años de observaciones. El procesamiento fue realizado con la ayuda del Sistema Integrado para el Manejo de Bases de Dados sobre Incendios Forestales (SIMBDIF) versión 1.2 (RODRÍGUEZ, 2002) y el Microsoft Excel.

La evaluación de la eficiencia se basó en los elementos metodológicos desarrollados por Rodríguez (1999). Se utilizaron los indicadores siguientes:

- Densidad de incendios: Es la cantidad de incendios que se producen por cada 1000 ha cubiertas de 
bosque (adaptado de MINISTERIO DE LA AGRICULTURA (MINAG), 1982).

- Densidad de área quemada: Es el área afectada por el fuego por cada 1000 ha cubiertas de bosque (adaptado de MINAG, 1982).

- Media del área quemadas por incendio: Es la relación entre el número de hectáreas quemadas y el número de incendios.

- Porcentaje de la superficie boscosa que se quema: Se expresa como la superficie quemada dividida por la superficie cubierta de bosques, multiplicado por 100.

- Tamaño de los incendios (clases de tamaño): Es el agrupamiento de los incendios según el área que han quemado. Existen varias clasificaciones de los incendios de acuerdo a su tamaño (RAMSEY; HIGGINS, 1981; OHARRIZ, 1991; DU MAROC, 2001). En este trabajo se ha utilizado la clasificación propuesta para Cuba por la Food and Agriculture Organization (FAO) (2001): incendios hasta 1 ha (manchón), de 1,01 a 4 ha (pequeño), de 4,01 a 40 ha (mediano), de 40,01 a 200 (grande) y encima de 200 ha (muy grande).

Todos los indicadores se evaluaron a través del periodo de años seleccionado, a través del año, según los tipos de cobertura forestal y según los grupos de especies. Los indicadores relacionados con el área cubierta de bosques se calcularon a partir de la superficie cubierta cada año para el caso de los análisis según el periodo de años y para el resto de los análisis se consideró la media de la superficie cubierta para los 10 años.

\section{RESULTADOS Y DISCUSIÓN}

\section{Análisis a través del periodo de años seleccionado}

En la tabla 1 se muestran los valores de los distintos indicadores de eficiencia del 2001 al 2010. Resulta interesante destacar que el valor medio de la densidad de incendios obtenida en el periodo para Monte Alegre es 4,29 veces superior al obtenido en Pinar del Río, no obstante, en esta región la densidad de área quemada y la media del área quemada media por incendio son superiores 6,91 y 24,54 veces respectivamente, a Monte Alegre, lo que indica mejor eficiencia en esta última región de forma general. No obstante, el porcentaje de la superficie boscosa que se quema es ligeramente superior en Monte Alegre.

Rodríguez y Soares (1998) obtuvieron para Pinar del Río en el periodo 1989-1996 valores medios menores que los obtenidos para esta región en el periodo que se analiza, con excepción de la densidad de incendios que fue ligeramente inferior, lo que indica que la eficiencia ha disminuido de un periodo a otro, a pesar de los esfuerzos organizativos realizados y la adquisición de herramientas manuales y de vehículos $4 \times 4$ para el transporte de las brigadas de forma rápida hasta lugares de difícil acceso. Estos resultados están relacionados con los altos valores obtenidos para todos los indicadores, excepto la densidad de incendios, durante el año 2006, cuya época de incendios fue antecedida de un largo periodo de sequía. Durante el periodo 1997-2006, Rodríguez et al. (2008) obtuvieron valores medios superiores para los indicadores de eficiencia analizados, lo cual estuvo vinculado a dos incendios de grandes proporciones ocurridos en 1999.

El valor de la media del área quemada por incendio obtenido en Monte Alegre es muy inferior a los reportados por Soares (1992) para Francia, Italia y España (1977-1982), Grecia (1978-1982), Chile (1964-1986), y Brasil (1983-1987). También fue inferior el valor obtenido en Pinar del Río comparado con los reportados para estos países y periodos con excepción de Francia. Madoui (2000) obtuvo en Argelia (1979-1987) un valor medio de 45,57 ha quemadas por incendios. Rodríguez (2000) obtuvo para Cuba (1984-1998) un valor medio de 15,00 hectáreas por incendios.

Según Soares (1992) mientras más eficiente sea la protección contra incendios en las áreas forestales, menor será la relación entre el área media quemada por año y el total del área protegida. Según este autor, la relación óptima en un eficiente sistema de protección debe quedar en torno al $0,05 \%$, pudiendo llegar hasta $0,10 \%$ en áreas de alto riesgo de ocurrencias o de vegetación extremadamente inflamable. De acuerdo con esto, el valor medio obtenido para el porcentaje de la superficie boscosa que se quema en ambas regiones no indica una buena eficiencia. No obstante, Rodríguez et al. (2008) obtuvieron para Pinar del Río (1997-2006) un valor de 0,54\%, superior a los obtenidos en este trabajo para las dos regiones objeto de estudio.

La distribución de los incendios según las clases de tamaño se presenta en la tabla 2. Se observa que en las dos primeras clases se ubica el 97,63 y el 74,97\% de los incendios en Monte Alegre y Pinar del Río respectivamente. Este resultado es similar a los reportados por Soares (1992) para Brasil (19831987), África del Sur (1985-1989) y Canadá (1969-1978). Rodríguez y Soares (2004) obtuvieron en 
Monte Alegre y en Pinar del Río (1998-2001) valores de 96,56 y 70,06\% respectivamente. Rodríguez et al. (2008) obtuvieron para Pinar del Río (1997-2006) un valor de 72,19\%.

Tabla 1. Indicadores de eficiencia en Monte Alegre (MA) y Pinar del Río (PR) (2001-2010).

Table 1. Efficiency indicators in Monte Alegre (MA) and Pinar del Río (PR) (2001-2010).

\begin{tabular}{lllllllll}
\hline & DI & \multicolumn{3}{c}{ DAQ } & & AQI & & PSBQ \\
\cline { 2 - 8 } & MA & PR & MA & PR & MA & PR & MA & PR \\
\hline 2001 & 0,5127 & 0,2200 & 0,1012 & 2,4832 & 0,1974 & 11,28 & 0,0101 & 0,2483 \\
2002 & 0,5282 & 0,0911 & 0,0901 & 1,1816 & 0,1707 & 12,97 & 0,0090 & 0,1182 \\
2003 & 0,6758 & 0,0771 & 0,1405 & 0,1791 & 0,2080 & 2,32 & 0,0141 & 0,0179 \\
2004 & 0,9710 & 0,1650 & 0,1885 & 1,6395 & 0,1942 & 9,94 & 0,0189 & 0,1639 \\
2005 & 0,9088 & 0,1205 & 0,0878 & 2,5684 & 0,0966 & 21,31 & 0,0088 & 0,2568 \\
2006 & 0,9166 & 0,2335 & 0,1799 & 8,8510 & 0,1963 & 37,90 & 0,0180 & 0,8851 \\
2007 & 1,0486 & 0,1467 & 0,2078 & 0,8113 & 0,1981 & 5,53 & 0,0208 & 0,0811 \\
2008 & 0,6447 & 0,1625 & 0,6255 & 0,9905 & 0,9702 & 6,10 & 0,0626 & 0,0991 \\
2009 & 0,0854 & 0,2609 & 0,0459 & 2,2600 & 0,5377 & 8,66 & 0,0046 & 0,2260 \\
2010 & 0,5981 & 0,1262 & 1,4112 & 0,3279 & 2,3594 & 2,60 & 0,1411 & 0,0328 \\
\hline Media & 0,6890 & 0,1604 & 0,3078 & 2,1292 & 0,4468 & 11,86 & 0,3078 & 0,2129
\end{tabular}

Densidad de Incendios (DI), Densidad de Área Quemada (DAQ), Media del Área Quemada por Incendio (AQI), Porcentaje de la Superficie Boscosa que se Quema (PSBQ).

Tabla 2. Distribución de los incendios por clases de tamaño en Monte Alegre y Pinar del Río (2001-2010).

Table 2. Forest fire distribution by size class in Monte Alegre and Pinar del Río (2001-2010).

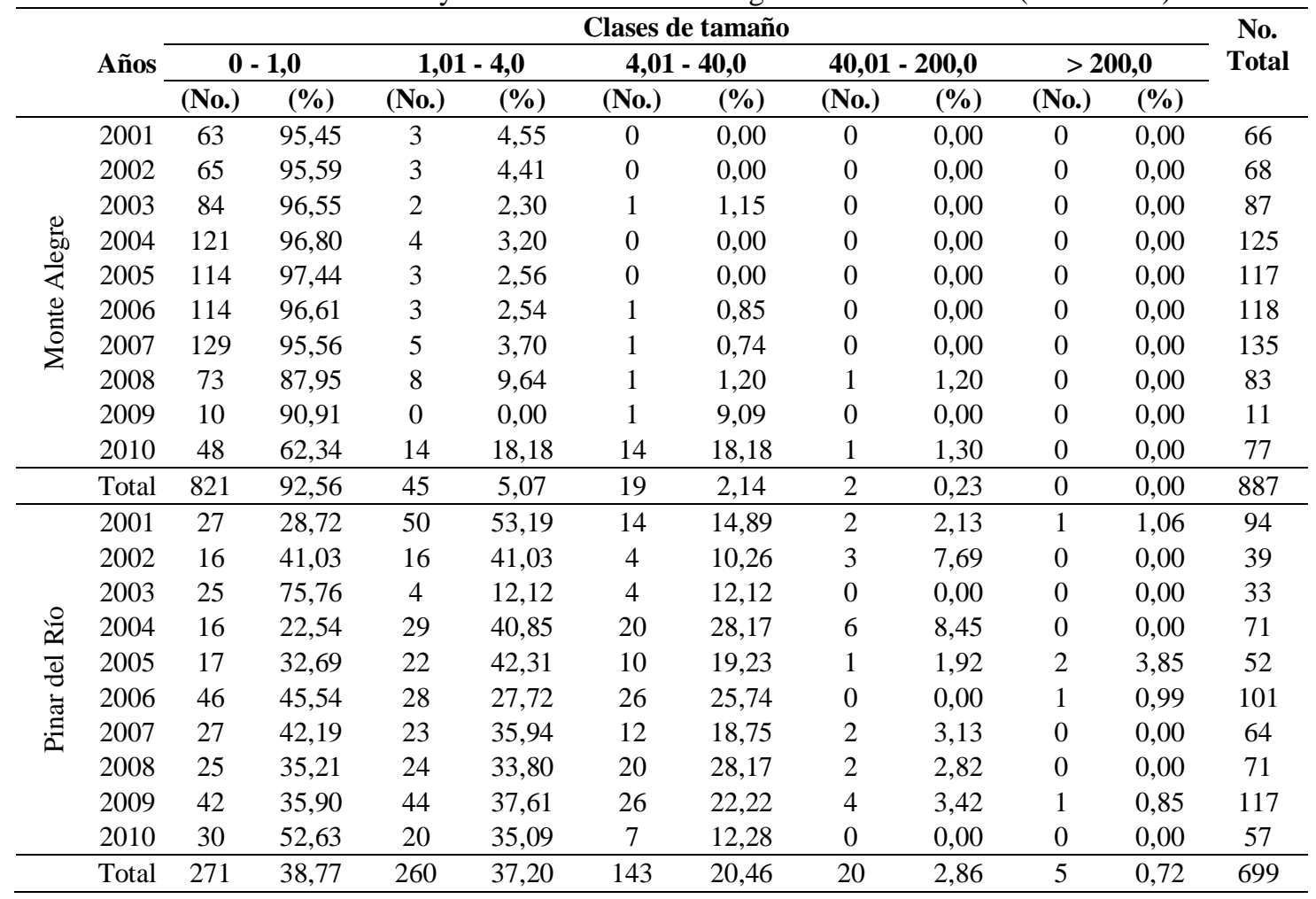

\section{Análisis a través del año}

En la tabla 3 se observa que en las dos regiones objeto de análisis la menor eficiencia del sistema de protección contra incendios, expresado por los altos valores obtenidos, se presenta durante la época de incendios: agosto-octubre en Monte Alegre y marzo-mayo en Pinar del Río, principalmente al final de la misma. En esta última región se presentaron valores altos también en octubre debido a un incendio de 
grandes proporciones ocurrido en ese mes, lo cual no es una regularidad. Estos resultados indican que aunque en sentido general la eficiencia de la protección es buena en ambas regiones, existen meses en los cuáles es preciso mejorar dicha eficiencia. Rodríguez (1999) y Rodríguez et al. (2008) obtuvieron en Pinar del Río durante los periodos 1990 - 1996 y 1997 - 2006 respectivamente, resultados similares.

Tabla 3. Indicadores de eficiencia en Monte Alegre (MA) y Pinar del Río (PR) por meses (2001-2010). Table 3. Efficiency indicators in Monte Alegre (MA) and Pinar del Río (PR) by month (2001-2010).

\begin{tabular}{lcccccccc}
\hline \multirow{2}{*}{ Meses } & \multicolumn{2}{c}{ DI } & \multicolumn{2}{c}{ DAQ } & \multicolumn{2}{c}{ AQI } & \multicolumn{2}{c}{ PSBQ } \\
\cline { 2 - 9 } & MA & PR & MA & PR & MA & PR & MA & PR \\
\hline Enero & 0,1165 & 0,0873 & 0,0194 & 0,4338 & 0,1668 & 4,97 & 0,0019 & 0,0434 \\
Febrero & 0,1709 & 0,1126 & 0,0159 & 1,8117 & 0,0932 & 16,09 & 0,0016 & 0,1812 \\
Marzo & 0,2796 & 0,2023 & 0,1058 & 1,3136 & 0,3785 & 6,50 & 0,0106 & 0,1314 \\
Abril & 0,2641 & 0,2459 & 0,0564 & 2,5518 & 0,2137 & 10,38 & 0,0056 & 0,2552 \\
Mayo & 0,5903 & 0,3631 & 0,0743 & 11,6474 & 0,1258 & 32,07 & 0,0074 & 1,1647 \\
Junio & 0,6680 & 0,1931 & 0,0879 & 0,8979 & 0,1316 & 4,65 & 0,0088 & 0,0898 \\
Julio & 0,6525 & 0,1471 & 0,0973 & 0,7265 & 0,1491 & 4,94 & 0,0097 & 0,0726 \\
Agosto & 1,3127 & 0,1310 & 0,7366 & 0,3902 & 0,5611 & 2,98 & 0,0737 & 0,0390 \\
Septiembre & 0,9710 & 0,0414 & 0,2946 & 0,2029 & 0,3034 & 4,91 & 0,0295 & 0,0203 \\
Octubre & 0,9166 & 0,0276 & 1,3589 & 1,0825 & 1,4826 & 39,25 & 0,1359 & 0,1083 \\
Noviembre & 0,4894 & 0,0230 & 0,1058 & 0,1074 & 0,2161 & 4,68 & 0,0106 & 0,0107 \\
Diciembre & 0,4583 & 0,0322 & 0,1255 & 0,0558 & 0,2739 & 1,74 & 0,0126 & 0,0056 \\
\hline Dicmyyyyyyyyy
\end{tabular}

Densidad de Incendios (DI), Densidad de Área Quemada (DAQ), Media del Área Quemada por Incendio (AQI), Porcentaje de la Superficie Boscosa que se Quema (PSBQ).

La distribución de los incendios según las clases de tamaño a través de los meses se presenta en la tabla 4. En correspondencia con los análisis realizados anteriormente, se observa que los porcentajes de incendios ubicados en las dos primeras clases de tamaño disminuyen durante los meses correspondientes a la época de incendios en ambas regiones. Esto reafirma la importancia de extremar las medidas de protección durante estos meses con el fin de alcanzar mejores resultados de eficiencia. En el caso de Pinar del Río se observa que varios incendio se ubican en clases mayores durante el mes de octubre, el cual no se incluye en la época de incendios, lo cual puede ser una llamada de atención para la toma de determinadas medidas organizativas también fuera de la época de incendios. Rodríguez et al. (2008), obtuvieron para Pinar del Río (1997-2006) resultados similares.

\section{Análisis según los tipos de cobertura forestal}

La tabla 5 muestra que los valores obtenidos en los indicadores que se analizan, con excepción de los correspondientes a la media del área quemada por incendios para Pinar del Río, son mejores en los bosques naturales que para los artificiales en ambas localidades. No obstante, los resultados indican que cuando en ellos ocurre un incendio se queman más hectáreas que en las plantaciones. Esto puede deberse a la accesibilidad hasta las áreas en que se encuentran unos y otros y a diferencias en cuanto a la ejecución de actividades de la silvicultura preventiva. Resultados similares obtuvieron Rodríguez et al. (2008) para Pinar del Río (1997-2006). También Rodríguez (1999) obtuvo en Pinar del Río (1990-1996) resultados similares, solo que en el caso de la media del área quemada por incendios el valor obtenido fue menor en los bosques naturales que en las plantaciones.

La tabla 6 muestra que el mayor porcentaje de incendios se agrupan en las dos primeras clases de tamaño, hasta 4 ha, lo cual es un buen indicador de eficiencia. No obstante, se observa que en Monte Alegre el porcentaje de incendios que se ubican en estas clases de tamaño es similar en ambos tipos de cobertura, mientras que en Pinar del Río hay diferencia entre los valores obtenidos en uno y otro tipo de cobertura vegetal, siendo superior el porcentaje en el caso de las plantaciones lo cual está en correspondencia con los análisis realizados anteriormente. De acuerdo con esto, el sistema de protección contra incendios en Pinar del Río es menos eficiente en el caso de los bosques naturales. Resultados similares encontraron Rodríguez et al. (2008) para Pinar del Río (1997-2006). 
Tabla 4. Distribución de los incendios por clases de tamaño en Monte Alegre y Pinar del Río por meses (2001-2010).

Table 4. Forest fire distribution by size class in Monte Alegre and Pinar del Río by month (2001-2010).

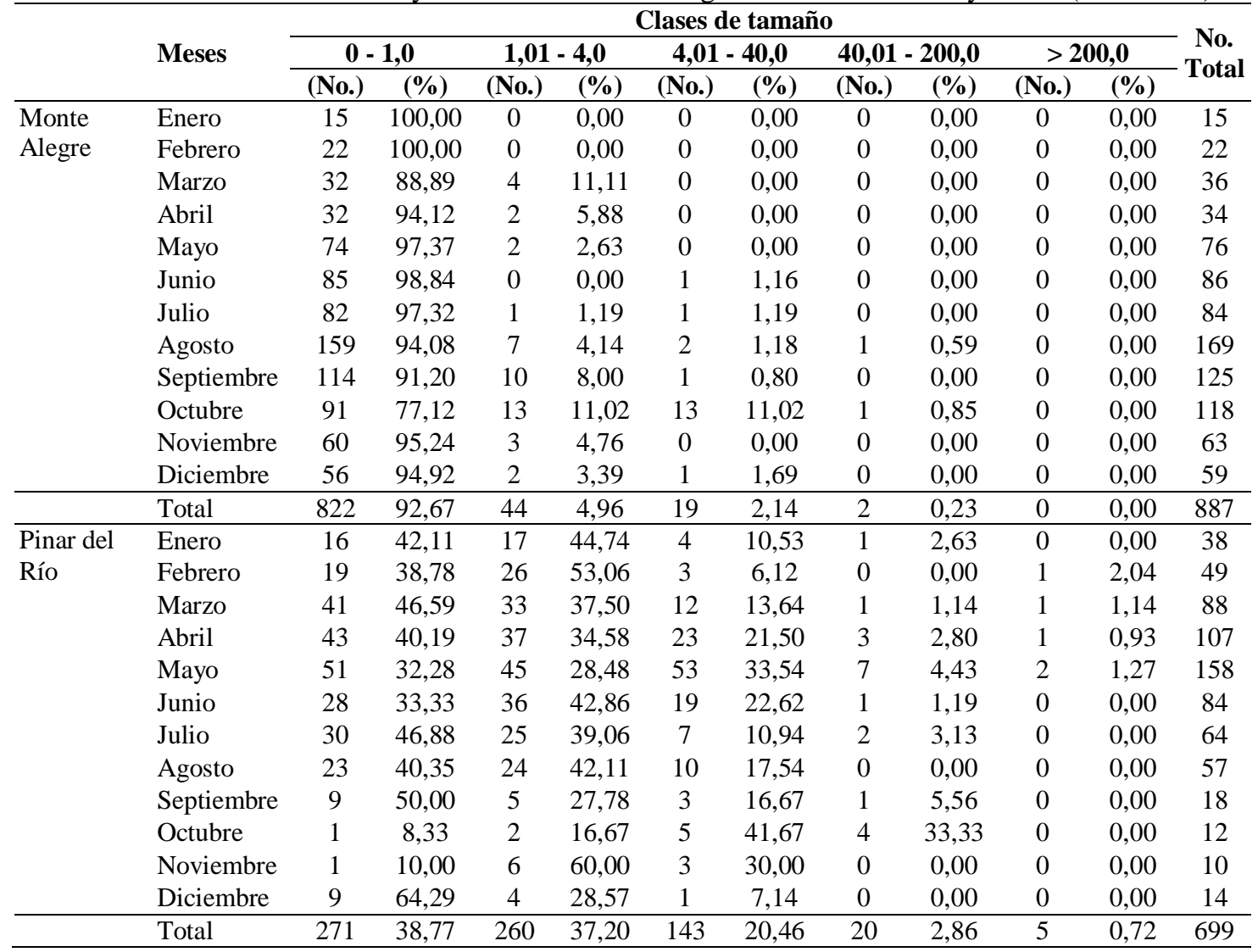

Tabla 5. Indicadores de eficiencia en Monte Alegre (MA) y Pinar del Río (PR) por tipos de cobertura forestal (2001-2010).

Table 5. Efficiency indicators in Monte Alegre (MA) and Pinar del Río (PR) by forest coverage (20012010).

\begin{tabular}{lcccccccc}
\hline Cobertura & \multicolumn{2}{c}{ DI } & \multicolumn{2}{c}{ DAQ } & \multicolumn{2}{c}{ AQI } & \multicolumn{2}{c}{ PSBQ } \\
\cline { 2 - 9 } vegetal & MA & PR & MA & PR & MA & PR & MA & PR \\
\hline Naturales & 1,5768 & 0,5586 & 0,6552 & 13,6646 & 0,4155 & 24,46 & 0,0655 & 1,3665 \\
Plantaciones & 4,7616 & 5,1371 & 1,9869 & 46,3866 & 0,4173 & 9,03 & 0,1987 & 4,6387 \\
\hline
\end{tabular}

Análisis según los grupos de especies

Los valores de los indicadores de eficiencia que se muestran en la tabla 7 muestran que en ambas regiones los mayores valores fueron obtenidos para el caso del Pinus spp. excepto para el indicador densidad de incendios en Pinar del Río, región en la cual el mayor valor se presentó en el Eucalyptus spp. Rodríguez (1999) obtuvo en Pinar del Río (1990-1996) los valores más altos de todos los indicadores para Eucalyptus spp., con excepción de la densidad de incendios que correspondió a la Casuarina spp. Rodríguez et al. (2008), encontraron para Pinar del Río (1997-2006) resultados similares a los obtenidos en este trabajo.

Los resultados obtenidos para las clases de tamaño de los incendios se muestran en la tabla 8 . Los porcentajes de incendios ubicados en las dos primeras clases de tamaño correspondientes a cada grupo de especies es menor en el caso del Pinus spp. en ambas regiones, lo cual indica menor eficiencia del sistema de protección contra incendios en este grupo de especies. Rodríguez et al. (2008), encontraron para Pinar del Río (1997-2006) resultados similares a los obtenidos en este trabajo. 
Tabla 6. Distribución de los incendios por clases de tamaño en Monte Alegre y Pinar del Río por tipos de cobertura forestal (2001-2010).

Table 6. Forest fire distribution by size class in Monte Alegre and Pinar del Río by forest coverage (2001-2010).

\begin{tabular}{|c|c|c|c|c|c|c|c|c|c|c|c|c|}
\hline & \multirow{3}{*}{$\begin{array}{c}\text { Cobertura } \\
\text { vegetal }\end{array}$} & \multicolumn{10}{|c|}{ Clases de tamaño } & \multirow{3}{*}{$\begin{array}{l}\text { No. } \\
\text { Total }\end{array}$} \\
\hline & & \multicolumn{2}{|c|}{0 - 1,0 } & \multicolumn{2}{|c|}{$1,01-4,0$} & \multicolumn{2}{|c|}{$4,01-40,0$} & \multicolumn{2}{|c|}{$40,01-200,0$} & \multicolumn{2}{|c|}{$>200,0$} & \\
\hline & & (No.) & $(\%)$ & (No.) & $(\%)$ & (No.) & $(\%)$ & (No.) & $(\%)$ & (No.) & $(\%)$ & \\
\hline Monte & Naturales & 194 & 95,57 & 5 & 2,46 & 3 & 1,48 & 1 & 0,49 & 0 & 0,00 & 203 \\
\hline \multirow[t]{2}{*}{ Alegre } & Plantaciones & 557 & 90,86 & 40 & 6,53 & 16 & 2,61 & 0 & 0,00 & 0 & 0,00 & 613 \\
\hline & Total & 751 & 92,03 & 45 & 5,51 & 19 & 2,33 & 1 & 0,12 & 0 & 0,00 & $816^{(*)}$ \\
\hline \multirow{3}{*}{$\begin{array}{l}\text { Pinar del } \\
\text { Río }\end{array}$} & Naturales & 61 & 32,62 & 61 & 32,62 & 53 & 28,34 & 8 & 4,28 & 4 & 2,14 & 187 \\
\hline & Plantaciones & 208 & 40,31 & 198 & 38,37 & 91 & 17,64 & 15 & 2,91 & 4 & 0,78 & 516 \\
\hline & Total & 269 & 38,26 & 259 & 36,84 & 144 & 20,48 & 23 & 3,27 & 8 & 1,14 & 703 \\
\hline
\end{tabular}

Nota: ${ }^{(*)} 71$ ocurrencias, que afectaron un área de 56,1769 ha, no informaron el tipo de cobertura vegetal afectada.

Tabla 7. Indicadores de eficiencia en Monte Alegre (MA) y Pinar del Río (PR) por grupos de especies (2001-2010).

Table 7. Efficiency indicators in Monte Alegre (MA) and Pinar del Río (PR) by the species group (2001-2010).

\begin{tabular}{lcccccccc}
\hline \multirow{2}{*}{ Grupos de especies } & \multicolumn{2}{c}{ DI } & \multicolumn{2}{c}{ DAQ } & \multicolumn{2}{c}{ AQI } & \multicolumn{2}{c}{ PSBQ } \\
\cline { 2 - 9 } & MA & PR & MA & PR & MA & PR & MA & PR \\
\hline Pinus spp. & 2,9206 & 3,6918 & 1,3582 & 66,4340 & 0,4650 & 18,00 & 0,1358 & 6,6434 \\
Eucalyptus spp. & 1,7400 & 10,1221 & 0,6060 & 45,7152 & 0,3483 & 4,52 & 0,0606 & 4,5715 \\
Casuarina spp. & 0,0000 & 1,7797 & 0,0000 & 7,6528 & 0,0000 & 4,30 & 0,0000 & 0,7653 \\
Otras especies & 0,1010 & 0,2678 & 0,0227 & 1,0204 & 0,2246 & 3,81 & 0,0023 & 0,1020 \\
Otra vegetación & 1,5768 & 0,0000 & 0,6552 & 0,0000 & 0,4155 & 0,00 & 0,0655 & 0,0000 \\
\hline
\end{tabular}

Tabla 8. Distribución de los incendios por clases de tamaño en Monte Alegre y Pinar del Río por grupos de especies (2001-2010).

Table 8. Forest fire distribution by size class in Monte Alegre and Pinar del Río by the species group (2001-2010).

\begin{tabular}{|c|c|c|c|c|c|c|c|c|c|c|c|c|}
\hline & \multirow{3}{*}{$\begin{array}{l}\text { Grupos de } \\
\text { especies }\end{array}$} & \multicolumn{10}{|c|}{ Clases de tamaño } & \multirow{3}{*}{$\begin{array}{l}\text { No. } \\
\text { Total }\end{array}$} \\
\hline & & \multicolumn{2}{|c|}{0 - 1,0 } & \multicolumn{2}{|c|}{$1,01-4,0$} & \multicolumn{2}{|c|}{$4,01-40,0$} & \multicolumn{2}{|c|}{40,01 - 200,0} & \multicolumn{2}{|c|}{$>200,0$} & \\
\hline & & (No.) & $(\%)$ & (No.) & $(\%)$ & (No.) & $(\%)$ & (No.) & $(\%)$ & (No.) & $(\%)$ & \\
\hline \multirow[t]{6}{*}{ Monte Alegre } & Pinus spp. & 340 & 90,43 & 22 & 5,85 & 14 & 3,72 & 0 & 0,00 & 0 & 0,00 & 376 \\
\hline & Eucalyptus spp. & 205 & 91,52 & 17 & 7,59 & 2 & 0,89 & 0 & 0,00 & 0 & 0,00 & 224 \\
\hline & Casuarina spp. & 0 & 0,00 & 0 & 0,00 & 0 & 0,00 & 0 & 0,00 & 0 & 0,00 & 0 \\
\hline & Otras especies & 12 & 92,31 & 1 & 7,69 & 0 & 0,00 & 0 & 0,00 & 0 & 0,00 & 13 \\
\hline & Otra vegetación & 194 & 95,57 & 5 & 2,46 & 3 & 1,48 & 1 & 0,49 & 0 & 0,00 & 203 \\
\hline & Total & 751 & 92,03 & 45 & 5,51 & 19 & 2,33 & 1 & 0,12 & 0 & 0,00 & 816 \\
\hline \multirow[t]{6}{*}{ Pinar del Río } & Pinus spp. & 172 & 37,80 & 162 & 35,60 & 99 & 21,76 & 17 & 3,74 & 5 & 1,10 & 455 \\
\hline & Eucalyptus spp. & 56 & 34,36 & 71 & 43,56 & 34 & 20,86 & 2 & 1,23 & 0 & 0,00 & 163 \\
\hline & Casuarina spp. & 1 & 50,00 & 1 & 50,00 & 0 & 0,00 & 0 & 0,00 & 0 & 0,00 & 2 \\
\hline & Otras especies & 42 & 53,16 & 26 & 32,91 & 10 & 12,66 & 1 & 1,27 & 0 & 0,00 & 79 \\
\hline & Otra vegetación & 0 & 0,00 & 0 & 0,00 & 0 & 0,00 & 0 & 0,00 & 0 & 0,00 & 0 \\
\hline & Total & 271 & 38,77 & 260 & 37,20 & 143 & 20,46 & 20 & 2,86 & 5 & 0,72 & 699 \\
\hline
\end{tabular}

\section{CONCLUSIONES}

La hipótesis de que la eficiencia del sistema de protección contra incendios es diferente en las dos regiones estudiadas se demostró parcialmente, lo cual puede ser comprobado por las conclusiones siguientes:

- Los valores medios obtenidos para los indicadores de eficiencia en el periodo 2001-2010 y la distribución de los incendios según sus clases de tamaño, muestran en sentido general, mejor eficiencia del sistema de protección contra incendios en Monte Alegre que en Pinar del Río. 
- En ambas regiones la eficiencia del sistema de protección contra incendios disminuye durante los meses correspondientes a la época de incendios: agosto-octubre en Monte Alegre y marzo-mayo en Pinar del Río.

- En Monte Alegre la eficiencia del sistema de protección contra incendios es similar en bosques naturales y en plantaciones, mientras que en Pinar del Río este sistema es menos eficiente en los bosques naturales.

- La eficiencia obtenida para el sistema de protección contra incendios tanto en Monte Alegre como en Pinar del Río es menor en el grupo de especies de los Pinus spp.

\section{REFERENCIAS}

ÇANAKÇIOGLU, H. Forest fire in Turkey. In: IUFRO WORLD CONGRESS, 19., 1990, Canada. Proceedings of... Canada: IUFRO, 1990. p. 26 - 41.

COMITÉ DE LUCHA CONTRA INCENDIOS FORESTALES (CLIF) - Libro rojo de la coordinación contra los incendios forestales. España: CLIF, 1997. 197 p.

DIRECCIÓN GENERAL DE CONSERVACIÓN DE LA NATURALEZA (DGCN). Los incendios forestales en España durante el decenio 1986-1995. España: Ministerio de Medio Ambiente; DGCN, 1996. $58 \mathrm{p}$.

DU MAROC, R. Forest fire situation in Morocco. International Forest Fire News, n. 25, p. 14 - 22, 2001.

FOOD AGRICULTURE ORGANIZATION (FAO) Técnicas de prevención de incendios forestales: informe final. La Habana, 2001. 61 p. Proyecto TCP/CUB/0066-FAO.

HAIGHT, R. G.; FRIED, J. S. Deploying wildland fire suppression resources with a scenario-based standard response model. INFOR, v. 45, n. 1, p. 31 - 39, 2007.

INSTITUTO DE PLANIFICACIÓN FÍSICA (IPF). Pinar del Río en cifras. Cuba: Instituto de Planificación Física, 1993 (Informe Plan Director de la Montaña).

LIMA, G. S.; SOARES, R. V. Avaliação da eficiência de combate aos incêndios florestais no Brasil. Revista Floresta, v. 22, n. 12, p. 25 - 38, 1992.

MAACK, R. Geografia física do estado do Paraná. Curitiba: Banco de Desenvolvimento do Paraná, 1968. $350 \mathrm{p}$.

MADOUI, A. Forest fire in Algeria and the case of the Domanial Forest of Bou-Taleb, Setif. International Forest Fire News, n. 22, p. 9 - 14, 2000.

MENDES, I. A theoretical economic model for choosing efficient wildfire suppression strategies. Forest Policy and Economics, v. 12, p. 323 - 329, 2010.

McCARTHY, G. J.; PLUCINSKI, M. P.; GOULD, J. S. Analysis of the resourcing and containment of multiple remote fires: the great divide complex of fires, Victoria, December 2006. Australian Forestry, v. 75 , n. 1 , p. $54-63,2012$.

MINISTERIO DE LA AGRICULTURA (MINAG). Norma ramal de la agricultura 555: incendios forestales términos y definiciones. Cuba: MINAG, 1982. 7 p.

NTAIMO, L.; GALLEGO ARRUBLA, J. A.; STRIPLING, C.; YOUNG, J.; SPENCER, T. A stochastic programming standard response model for wildfire initial attack planning. Can. J. For. Res., v, 42, p. 987 - 1001, 2012.

NUNES, J. R. S.; BATISTA, A. C.; SOARES, R. V.; FIER, I. S. N. Climatologia do comportamento da precipitação no Distrito Florestal de Monte Alegre. Floresta, v. 39, n. 4, p. 783 - 792, 2009.

NUNES, J. R. S.; FIER, I. S. N; SOARES, R. V.; BATISTA, A. C. Desempenho da Fórmula de Monte Alegre (FMA) e da Fórmula de Monte Alegre Alterada (FMA+) no Distrito Florestal de Monte Alegre. Floresta, v. 40, n. 2, p. 319 - 326, 2010. 
OHARRIZ, S. Protección contra incendios forestales. Editorial Pueblo y Educación, 1991. 76 p.

RACHANIOTIS, N. P.; PAPPIS, C. Scheduling fire-fighting tasks using the concept of "deteriorating jobs". Can. J. For. Res., v. 36, p. 652 - 658, 2006.

RAMSEY, G. S.; HIGGINS, D. G. Canadian forest fire statistics. Ontario: Canadian Forestry Service, 1981. 71 p. Information Report PI-X-9.

RODRÍGUEZ, M. P. R. Bases metodológicas para el perfeccionamiento de la prevención de los incendios forestales. 100 p. Tesis (Doctorado en Ciencia Forestales) - Universidad de Pinar del Río, Cuba. 1999.

. An overview about forest fires in Cuba. International Forest Fire News, n. 22, p. 20 - 23, 2000.

Sistema Integrado para el Manejo de Bases de Datos sobre Incendios Forestales (SIMBDIF) versión 1.2. In: CONGRESO FORESTAL VENEZOLANO, 3., 2002. Actas del... Venezuela, 2002.

RODRÍGUEZ, M. P. R.; REINA, J. M. C.; HERRERA, L. A. O. Eficiencia de la protección contra incendios forestales en Pinar del Río. Revista Forestal Baracoa, v. 27, n. 2, p. 31 - 37, 2008.

RODRÍGUEZ, M. P. R.; SOARES, R. V. Comportamiento histórico de los incendios forestales en la provincia de Pinar del Río, Cuba. Revista Floresta, v. 28, n. 1/2, p. 03 - 18, 1998.

. Análisis comparativo entre los incendios forestales en Monte Alegre, Brasil y Pinar del Río, Cuba. Revista Floresta, v. 34, n. 2, p. 101 - 107, 2004.

RODRÍGUEZ, M. P. R.; SOARES, R. V.; BATISTA, A. C.; TETTO, A. F.; BECERRA, L. W. M. Comparação entre o perfil dos incêndios florestais de Monte Alegre, Brasil, e de Pinar del Río, Cuba. Floresta, v. 43, n. 2, p. 231 - 240, 2013.

SOARES, R. V. Determinação de um índice de perigo de incêndio para a região centro-paranaense, Brasil. 72 f. Dissertação (Mestrado em Ciências) - Centro Tropical de Ensino e Investigação, Instituto Interamericano de Ciências Agrícolas OEA, Turrialba, Costa Rica, 1972.

. Incêndios florestais: controle e uso do fogo. Curitiba: FUPEF, 1985. 213 p. $121,1988$.

Perfil dos incêndios florestais no Brasil, de 1984 a 1987. Revista Floresta, v. 22, n. 12, p. 94 $\overline{1992 .}$

. Ocorrência de incêndios em povoamentos florestais. Revista Floresta, v. 22, n. 12, p. 39 - 53,

Estatísticas dos incêndios florestais no Brasil. In: SOARES, R. V.; BATISTA, A. C.; NUNES, J. R. S. Incêndios florestais no Brasil: o estado da arte. Curitiba, 2009. p. 1 - 20.

TETTO, A. F.; BATISTA, A. C.; SOARES, R. V. Ocorrência de incêndios florestais no estado do Paraná, no período de 2005 a 2010. Floresta, v. 42, n. 2, p. 391 - 398, 2012a.

TETTO, A. F.; SOARES, R. V.; BATISTA, A. C.; WENDLING, W. T. Eficiência do combate aos incêndios florestais, no período de 1965 a 2009, na Fazenda Monte Alegre, Paraná. Scientia Forestalis, v. 40, n. 96 , p. 483 - 489, 2012 b.

VÉLEZ, R. Los incendios forestales en el Mediterráneo: perspectiva regional. Revista Unasylva, v. 41, n. 162 , p. $3-9,1990$.

VENTURI, N. L.; ANTUNES, A. F. B. Determinação de locais ótimos para implantação de torres de vigilância para detecção de incêndios florestais por meio de sistema de informações geográficas. Floresta, v. 37, n. 2, p. 159 - 173, 2007.

WADSWORTH, F. H. Producción forestal para América Tropical. Washington: Departamento de Agricultura de los EE.UU. USDA, 2000. 397 p. Manual de Agricultura. 
